Gazi University
Journal of Science
http://dergipark.gov.tr/gujs

\title{
Deep Learning-Based Architectures for Recognition of Cow Using Cow Nose Image Pattern
}

\author{
Rotimi-Williams BELLO*(D), Abdullah Zawawi Hj TALIB (D) , Ahmad Sufril Azlan Bin MOHAMED (D) \\ School of Computer Sciences, Universiti Sains Malaysia, 11800, Pulau, Pinang, Malaysia
}

\author{
Highlights \\ - Deep learning approach for learning discriminating texture features of cow nose image. \\ - Deep learning-based methods enhance animal biometrics. \\ - Stacked denoising auto-encoder for encoding and decoding extracted features. \\ - Deep belief network for learning and representing features. \\ - Deep belief network has $98.99 \%$ accuracy compared to other methods.
}

\begin{tabular}{l} 
Article Info \\
\hline Received: $16 / 08 / 2019$ \\
Accepted: $12 / 02 / 2020$ \\
Keywords \\
\hline Animal biometrics \\
Deep learning \\
Cow nose image \\
SDAE \\
DBN
\end{tabular}

\begin{abstract}
Stacked denoising auto-encoder and deep belief network are proposed as methods of deep learning for cow nose image texture feature extraction, and for learning the extracted features for better representation. While stacked denoising auto-encoder is applied for encoding and decoding of the extracted features, a deep belief network is applied for learning the extracted features and representing the cow nose image in feature space. Stacked denoising auto-encoder and deep belief network help in animal biometrics. Biometrics emanated from computer vision and pattern recognition and it plays an important role in the automated animal registration and identification process. Using the visual attributes of cow, and for the fact that the existing visual feature extraction and representation methods are not capable of handling cow recognition; deep belief network and stacked denoising auto-encoder are proposed. An experiment performed under different conditions of identification indicated that deep belief network outshines other methods with approximately $98.99 \%$ accuracy. 4000 cow nose images from an existing database of 400 individual cows contribute to the community of research especially in the animal biometrics for identification of individual cow.
\end{abstract}

\section{INTRODUCTION}

For any successful animal husbandry, it is important to have reliable, affordable, scalable and effective livestock management for significant animal performance. Herders in the country face a lot of challenges such as cow rustling due to the nomadic system of grazing which has greatly reduced productivity and profit. The manual identification approach for cow recognition for a long time had posed a difficulty for herders and animal husbandry community in monitoring cows, and the existing cow tracking methods are not accurate enough such as in the case where some external factors such as background patches might distort the images and contribute to the increasing difficulty of the detection process $[1,2]$. Hence, there is a need for proper monitoring of cow using recent methods for reliable tracking, recognition, and identification of individual cow in herds. What the animal looks like as a consequence of the interaction of its genotype and the environment is made up of an organism's observable structural features [3, 4]. Though, the manual framework methods of identification provide traditional methods for individual cow identification in the herd but, they are incapable to provide satisfactorily, the security level for herders and cow breeders in monitoring cow all over the world. Biometrics applications in monitoring animals emanated from computer vision and pattern recognition which are branches of artificial intelligence [3,5]. 
Different animal monitoring and identification methodologies such as global positioning system (GPS), virtual fencing, using of tattoo, tags, radio frequency identification (RFID), sensors, photographs, drawings, descriptions, branding (hot and freeze), ear notching, microchip implants, iris, and retina scan, and muzzle or nose prints are known animal recognition and identification methods [1]. The main problems with some of these methodologies are their low image quality, injury-induced on animal body parts, low-frequency coverage, easily lost tags, among others. A RFID chip-based techniques, which most of the time is embedded in the animal body is used for tracking and identification [1, 3, 5]. But, according to Wang et al. [6] and Krizhevsky et al. [7], the different layers possess by deep learning framework enables the modeling and representation of the difficult data variation for the sake of recognizing the animal. A cow recognition system that is based on enhanced deep learning approaches and frameworks is proposed to solve the recognition and identification problems known with the traditional identification methods by learning the nose image pattern, giving the feature proper representation and classifying them jointly for a specific task $[8,9]$.

According to Wang et al. [6], RFID faces a lot of challenges because of its implementation and management of its protocols and chips at different points. Deep learning, an emerging field under computer vision is an approach employ recently by researchers for the recognition and detection of what the animal looks like as a consequence of the interaction of its genotype and the environment. Deep learning is generally accepted as one of the most recent, reliable and accurate techniques for the extraction of features and for the individual animal representation $[1,10]$. The technology behind deep learning enables its framework to perform learning on the extracted sets of biometrics species feature for the purpose of representing and identifying the species $[8,11]$.

This paper addresses the problem of cow recognition based on biometrics features of the cow nose pattern captured on image using deep belief network (DBN) and stacked denoising auto-encoder (SDAE) deep learning-based recognition architectures. The pattern of the cow nose image is made up of robust features of texture which the proposed cow recognizing system recognizes using the frameworks of deep learning. After the cow recognition system has captured the image of the cow nose pattern, the captured image is made to undergo pre-processing to remove the background patches, noises, and other discriminatory particles using Gaussian filtering techniques and then employ deep learning techniques for the identification of the individual cow.

This research work is primarily for the identification of cow to mitigate cow rustling, and our literature findings revealed no previous works that used deep learning approach for the identification of cow using animal biometrics characteristic features (nose image pattern) based on deep learning approach utilizing the existing database of cow nose images owned by combined agriculture, forestry and wildlife ministries in Nigeria. The remainder of this work is as follows: presented in section two is the research related work; methods and materials employed to achieve the research objectives are presented in section three; while the results and discussion are presented in section four; section five concludes the research.

\section{LITERATURE REVIEW}

Due to more and rapid growth experienced in the application of biometrics technological systems in detecting, tracking, recognizing, identifying, and monitoring individual animals; so many researchers have developed an interest in the field and more efforts are still being put into improving it [3, 12-19]. One of the recently developed methods is a convolutional neural network (CNN) which is one of the main approaches used in deep learning for image recognition and classifications. The CNN framework is built in such a way that the image classifiers accept a cow nose image as input, process the image and classify the image under a particular category via the multiple convolutional layers and pooling layers. The extraction of the cow nose features is directly from the cow nose image which is seen as an array of pixels. Technically, the essence of modeling $\mathrm{CNN}$ is to train and test each cow nose image inputed which will go through it via a series of convolutional layers with pooling, filters, softmax function and fully connected layers for the classification of cow nose image with probability values in the range of 0 and 1 [14, 15]. Other methods are stacked denoising auto-encoder which is applied to encode and decode the extracted features, and a 
deep belief network which is applied for learning the extracted features and representing the cow nose image in feature space [18].

The physical visual characteristics such as the nose image possess by the target species are what the animal biometrics-based recognition system uses for the identification of an individual animal. Basically, for animal identification, there are two known recognition methods in literature which are employed for identifying cow. The application of the first recognition method leaves on the animal's body a permanent mark, and temporary mark is left on the animal's body using the other recognition method. Instances of permanent mark on the animal's body for an individual animal identification including the limitations are put together in both early and recent works on animal biometrics $[2,15,17]$. Among these popular invasive methods for animal identification with permanent mark on the animal's body are the ears tattooing, ears tagging, microchips embedding and branding with health implications, namely mild sepsis, hemorrhaging, and other animal infections $[15,17]$.

Instances of temporary mark on the animal's body for an individual animal identification including the limitations are addressed in the animal biometrics work of Barron et al. [11]. As earlier iterated, the application of RFID for animal identification is the most promising classical identification technique. Furthering their research, Minagawa et al. [20] presented a cow identification framework using nose images. On white A-5 paper were the nose images captured with conspicuous ink and the performance evaluation of the proposed approach was made by using filtering techniques. The authors employed the processes of binary transformation and structural approaches to analyze the nose image, and they reported 0.419 equal error rate (EER).

Similar to Minagawa et al. [20], was the technique proposed by Barry et al. [21] for recognition of cow using nose image. Experimentation carried out by them in order to evaluate the performance of the proposed approach produced 241 false non-match rates (False NMR) over 560 genuine acceptance rate (GAR), and 5197 false matches (FM) over 12,160 impostors closely matching with the same 0.419 ERR value respectively. According to Nasirahmadi et al. [5], the feasibleness of employing Delaunay triangulation and image processing methods for the change detection in pigs group lying behavior under farm business conditions and how the changes help in understanding the environmental factors that affect both the pigs' welfare and health conditions was investigated.

Scale-invariant feature transform (SIFT) descriptor similar to rectangular gradients histogram (R-HOG) [22] approach was used for the cow recognition framework proposed by Awad et al. [23] to localize and to detect the region of interest (ROI) in the nose images for the cow identification in database of 90 nose images $(6 \times 15=90)$. The application of the SIFT keypoint matching based descriptor was employed by them for the matching of the nose images. RANSAC technique was used with the SIFT in order to mitigate the noises such as outliers points for better identification. Nonetheless, the results of the experiment of the proposed approach have no cross-validation; there was poor identification accuracy from the noises such as outliers, poor quality of image, and blurriness.

Similar to the work of Awad et al. [23] is the work found in Noviyanto and Arymurthy [24], the author proposed a matching enhancement technique in SIFT descriptor approach for the recognition of cow in 160 nose images database $(4 \times 4 \times 10=160)$ and the performance of the matching enhancement technique was compared to the approach of the original SIFT with equivalent ERR value of 0.0167. Proposed in Kumar et al. [25] is a framework to ascertain the identity of individual cows based on the image of their face. They used the extracted features of the face to evaluate the performance of their proposed system.

Ehsani et al. [26] employed the use of image processing techniques and computer vision-based techniques to model visually intelligent agents, their model takes visual information as input and the actions of the agent were directly predicted. In this work, they introduced a DECADE, which is an ego-centric video dataset from a dog's perspective. How the dog moves and acts were modeled using the data. But, their work was limited to considering only visual data, even though they testify by themselves to the fact that intelligent agents employ a variety of input modalities such as sound, touch, smell and so on when interacting with the world. They also limited their work to the modeling of a specific and single dog. Data could be collected from multiple dogs for generalized evaluation across dogs. Gaber et al. [27] proposed a nose image-based 
cow recognition approach for local texture features extraction from the nose images using algorithm of local binary pattern (LBP), a local texture descriptor based technique.

\section{METHODS AND MATERIALS}

The methods used in this section were motivated by the drawbacks of the previous animal recognition system and the need to develop an enhanced automated cow recognition method based on the biometrics system that will be robust and non-invasive for the identification of cow.

\subsection{System Requirements}

The designed system for recognizing and identifying cow can operate on any computer that has Windows as the operating system. In developing the system, the specifications for the computer hardware are as follows

(a) Operating Systems

- Windows 10

(b) Processing Device

- Minimum specification: Any Intel or AMD x86-64 processing unit

- Recommended specification: Any Intel or AMD x86-64 processing unit with 4 logical cores and AVX2 instruction set support

(c) Disk

- Minimum specification: HDD of 3.1 GB in space for MATLAB only, 5-8 GB in space for a typical installation and $750 \mathrm{~GB}$ of hard disk space for storage

- Recommended specification: An SSD is recommended

(d) RAM

- Minimum specification: 4GB (including 2GB RAM dedicated for visualization)

- Recommended specification: 8GB

(e) Graphics

- Minimum specification: Does not require any specific graphics card

- Recommended specification: Accelerated graphics card that supports OpenGL 3.3 with 1 GB GPU memory.

\subsection{Software Requirements}

MATLAB R2019b toolbox for image processing and computer vision is required for the experiment. To develop the complete system, personal computer running operating system Microsoft Windows 10 with the specifications iterated in section 3.1 is employed. Microsoft Visual Studio 2019 and MATLAB R2019b toolbox for image processing and computer vision are included for optimised experiment. MATLAB $\mathrm{R} 2019 \mathrm{~b}$ provides image processing and computer vision Toolbox ${ }^{\mathrm{TM}}$ through which a set of algorithms and workflow applications are provided for the processing and visualization of images, and for algorithm development. This work optimises MATLAB R2019b in the application of object segmentation, and object identification using deep learning techniques. Ground truth labeling and calibration of workflows are automated by computer vision applications. $\mathrm{C} / \mathrm{C}++$ code generation is supported by many MATLAB $\mathrm{R} 2019 \mathrm{~b}$ toolbox functions for desktop prototyping and embedded vision system deployment which is employed in this work.

Presently, the available cow nose image pattern database cannot be employed for the evaluation of the reigning state-of-the-art based recognition and identification algorithms meant for the recognition and identification of cow using cow nose image pattern. To cater for these issues, the existing database which consists of 4000 cow nose images from 400 individual cows (subject) captured using a high-resolution 
camera (thirty megapixels) by the ministries of agriculture, forestry, and wildlife, Nigeria was utilized for the identification process.

Gaussian filtering technique is the main filtration technique employed for this work, and finding the difference between two Gaussian functions produces the difference of the Gaussian filter [28]. This is calculated by the application of two Gaussian operators with different values of $\sigma$ to an image and their differences generated smoothened images. The expression of Gaussian's difference is

$a(b, c)=a_{1}(\mathrm{~b}, \mathrm{c})-a_{2}(\mathrm{~b}, \mathrm{c})$

where $a_{1}(\mathrm{~b}, \mathrm{c})$ and $a_{2}(\mathrm{~b}, \mathrm{c})$ are two Gaussian functions

$a_{1}(\mathrm{~b}, \mathrm{c})=\mathrm{e}^{r 2 / 2 \sigma_{1} 2}$

$a_{2}(\mathrm{~b}, \mathrm{c})=\mathrm{e}^{r 2 / 2 \sigma_{2} 2}$

where $\sigma 1>\sigma 2$

From Equations (1)-(3)

$a(\mathrm{~b}, \mathrm{c})=\frac{r^{2}}{\mathrm{e}^{2 \sigma_{1} 2}}-\frac{r^{2}}{\mathrm{e}^{2 \sigma_{2} 2}}$

Shown in Figure 1 are samples of nose images of the cow from the database. By applying Kumar et al. [25], the images were filtered to remove from the nose images blurriness, effects of low illumination and background patches.
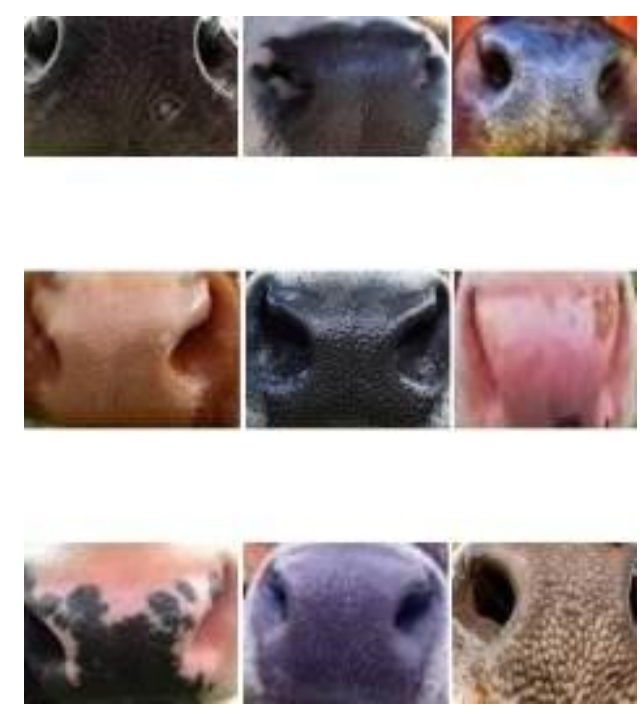

Figure 1. Some nose images from database

To monitor the traceability, health information, and performance of cows whether in-housed or out-housed, smart devices such as digital cameras and smart cameras have helped in capturing so many images used in the biometrics processes. A camera of high-resolution (thirty megapixels) was employed in capturing the nose image of the cow for the individual cow identification with the use of the proposed cow recognition system. The idea behind human fingerprint image capturing and analysis for human recognition and identification was employed in the cow nose image capturing for recognition and identification of cow because the minor points in the human fingerprint pattern are similar to the cow nose print patterns which are robust and full of rich dense texture feature. 
One of the discriminatory features of the cow nose image pattern, beads image features consists of an image pattern of anon-uniform in the cow nose images. The ridges' image features are the image patterns that are uniform which is very synonymous with the ridges found on the human fingerprint images. Shown in Figure 2 is the database that contains blurred nose image patterns caused by the poorly lit environment and cow postures resulting to low-in-quality images.

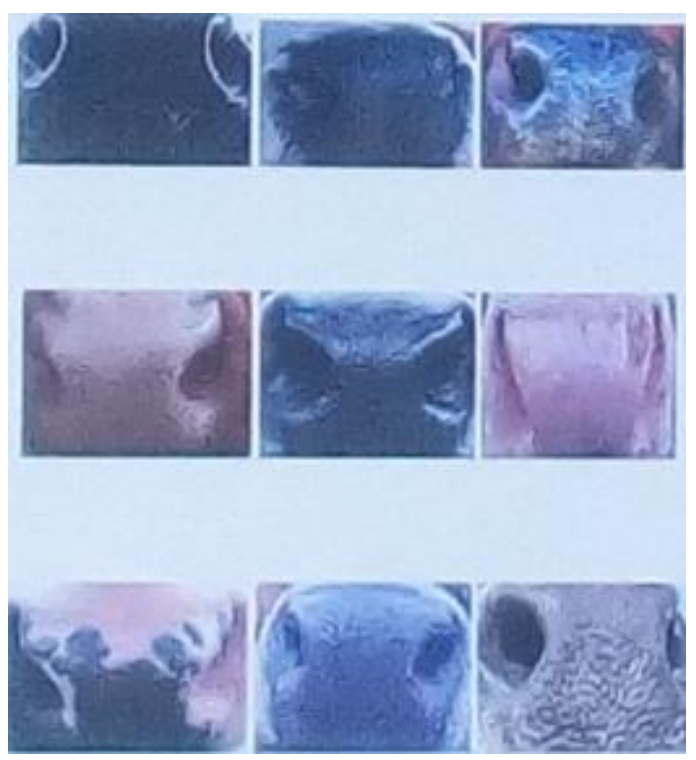

\section{Figure 2. Blurred and poor illumination of nose images}

Based on the similarity that exists between the human fingerprint and cow nose print, and using the robust features of the nose images database, we proposed a novel method for recognizing and identifying cow based on the nose image characteristics with the use of deep learning convolutional neural network (DL$\mathrm{CNN}$ ) architecture. Utilized for the experiments, is an existing database of cow nose which consists of 4000 cow nose images of 400 individual cows (subject) from which the discriminatory texture nose features of the beads and the ridges were extracted in pre-processing steps as shown in Figure 3, while Figure 4 shows the colored image of the cow nose with beads and ridges.

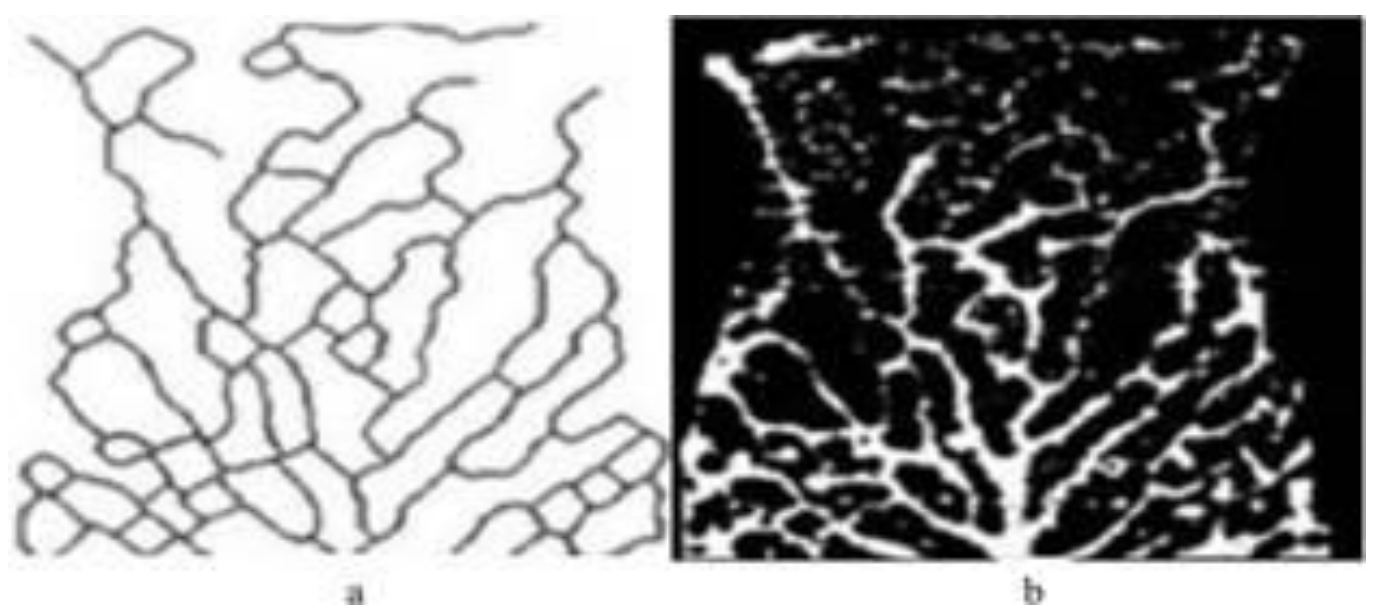

Figure 3. (a) Conversion of image to Gaussian blur image (background subtraction) (b) Filtration of discriminatory features

One of the main advantages of the difference of Gaussian as a filtering technique (Figure 3a) is the ability to use it for both gray and color images. 


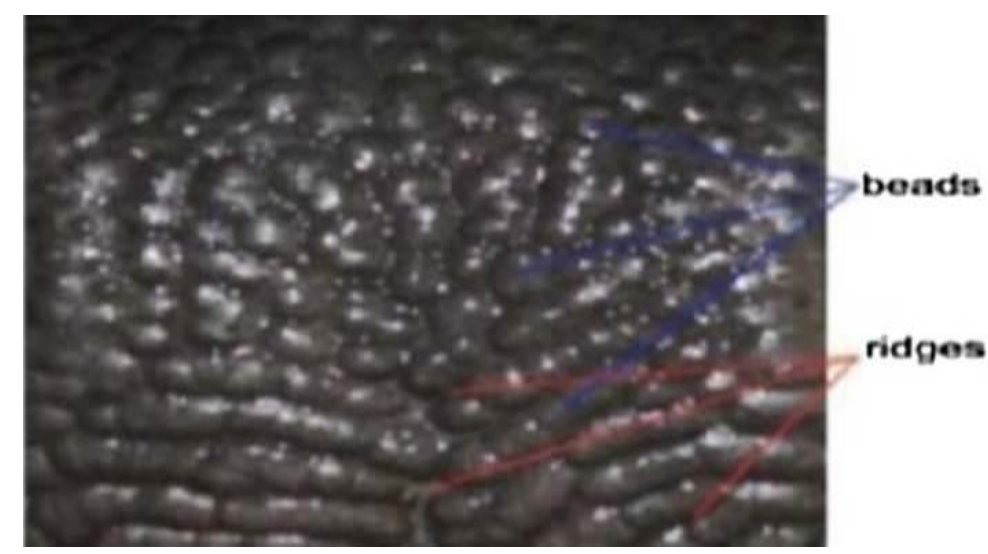

Figure 4. Colored image of cow nose with beads and ridges

In enhancing the identification process, different pre-processing techniques that are applicable to animal images were made use of in order to get the patches removed and noises reduced from the captured cow nose images. Low illumination and poor image quality are two of the most fundamental challenges militating against image capturing and processing especially cow nose image. In order to remove the patches, noises and other heterogenous objects captured with the cow nose images during data capturing, the images were transformed to grayscale images [28]. Figure 5 shows the framework of the deep learning that was used for the proposed cow recognition system.

The captured cow nose images in their color form are passed to the pre-processing technique which accepts and converts the images to grayscale prior to conveying them into the filter for patches and noises removal. To generate the desired output, the process of extracting the features involves performing convolutional and pooling procedure on the images until the classfier stage reaches where the images are processed for classification analysis. An auto-encoding method was used for the removal of the noises. The auto-encoding technique is primarily employed to encode and decode the extracted features [29]. The two primal auto encoder components are the encoder and the decoder. For example, if (A) is an input, an encoder maps it to the nodes in the hidden layer using a function that is called deterministic mapping function ( $\mathrm{f}: \mathrm{h}=\mathrm{f}(\mathrm{A})$ ) as shown in Equations (5) and (6)

$f=G \theta A=s(w \cdot A+\Delta)$

where

$\theta=(w, \Delta)$ is the parameter set, s represents the sigmoid, (w) is $\alpha \times \alpha$ weight matrix. $\Delta$ is the offset vector of size $\alpha^{\prime}$. To map the feature vector a' of $\alpha$ dimension, using a decoder function $G^{\prime} \theta$, feature $\mathrm{f}$ is applied such that,

$Y^{\prime \prime}=G^{\prime} \theta^{\prime} f=s\left(w \cdot A^{\prime}+\Delta^{\prime}\right)$

where the decoder parameter $\theta=\left(w^{\prime}, \Delta^{\prime}\right)$ is set. 


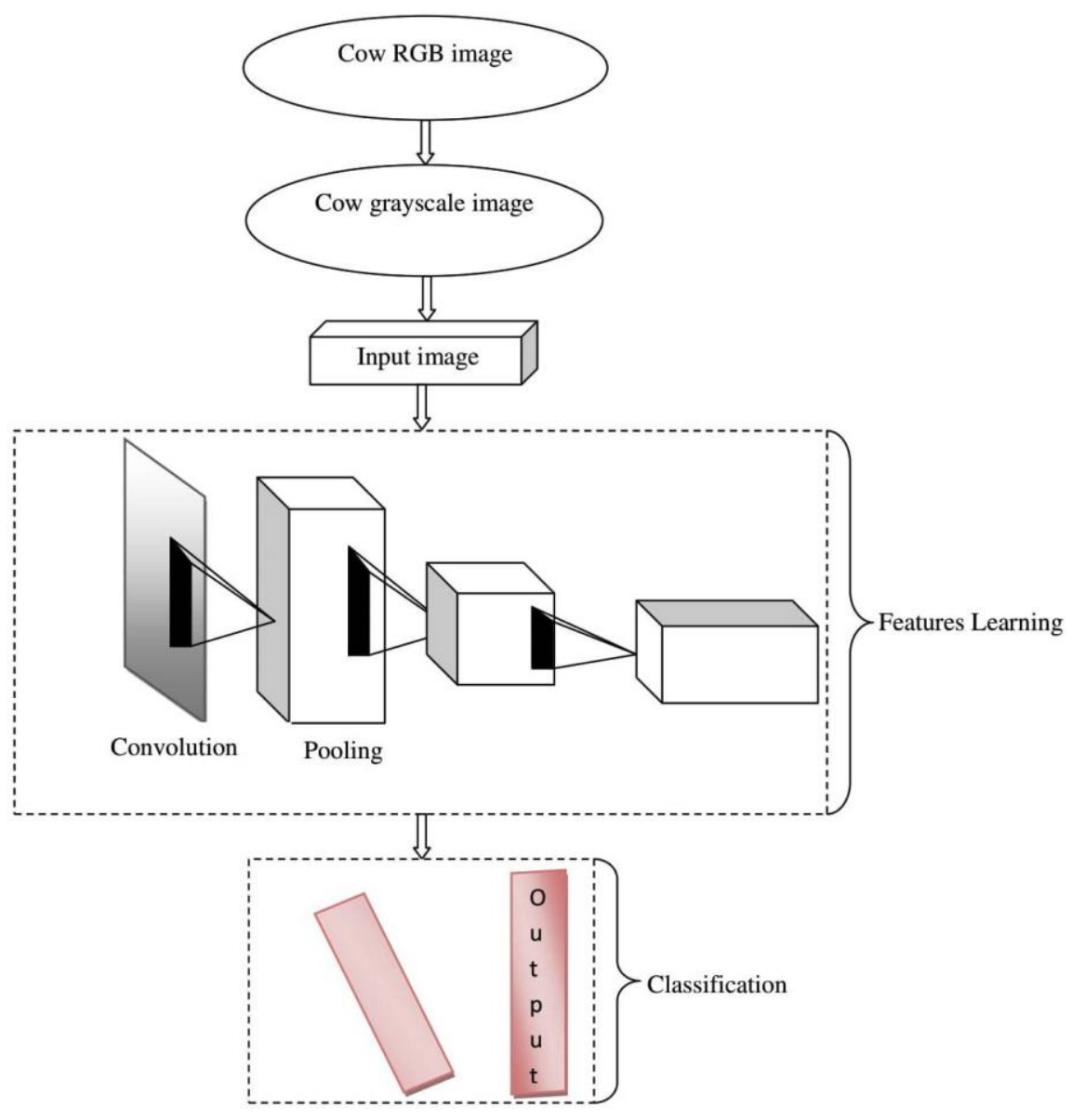

Figure 5. Neural network with convolutional layers for cow recognition

Technically, the essence of modeling CNN is to train and test each cow nose image inputed which will go through it via a series of convolutional layers with pooling, filters, softmax function and fully connected layers for the classification of cow nose image with probability values in the range of 0 and $1[14,15]$. Convolutional layer is the layer that comes first in the arrangement of layers for cow nose features extraction as shown in Figure 5. By using squares of input data to learn the cow nose image features, convolution principally conserves the relationship existing between pixels. This involves image matrix and a filter as two inputs of a mathematical operation applied in its execution as shown in Equations (1) to (6) . Pooling layers principally reduce the parameter's number (dimensionality size) when the available images are too large.

\section{RESULTS AND DISCUSSION}

The results and the evaluation of the identification accuracy of the cow recognition algorithm are presented and discussed in this section having carried out the experimental procedure for the training and the testing of the proposed model effectuality using the nose image pattern of the cow. For the evaluation of the identification accuracy in a proliferation settings, the recognition algorithm of the proposed approach was compared to other recognition algorithms. The acquired dataset is divided into two sets, namely the training dataset and the testing dataset. 100 nose images $(10$ cows (subject $) \times 10$ images (each subject)) were used as training dataset. 300 pairs of testing (30 cows (subject) $\times 10$ images (each subject)) were used as testing dataset.

To evaluate performance, the technique of local feature descriptor is applied for efficient extraction and encoding of cow nose image texture features, though, in this paper, handcrafted texture feature descriptor techniques were used for extracting the texture features of beads and ridges from the cow nose image. As earlier iterated, to mitigate the external factors such as low illumination, background patches and poor 
image quality that affect the captured images, the process of normalization and the descriptor is important. To perform the neccessary tasks that are involved in the normalization and the descriptor process, there's conversion of cells to blocks. In the course of this process, there was overlapping of blocks and sharing of cells among the blocks which were normalized separately.

Comparing SDAE approach to other approches as shown in Figures 6 and 7, the highest percentage of corrupted images was revealed using SDAE making it suitable for cow recognition.
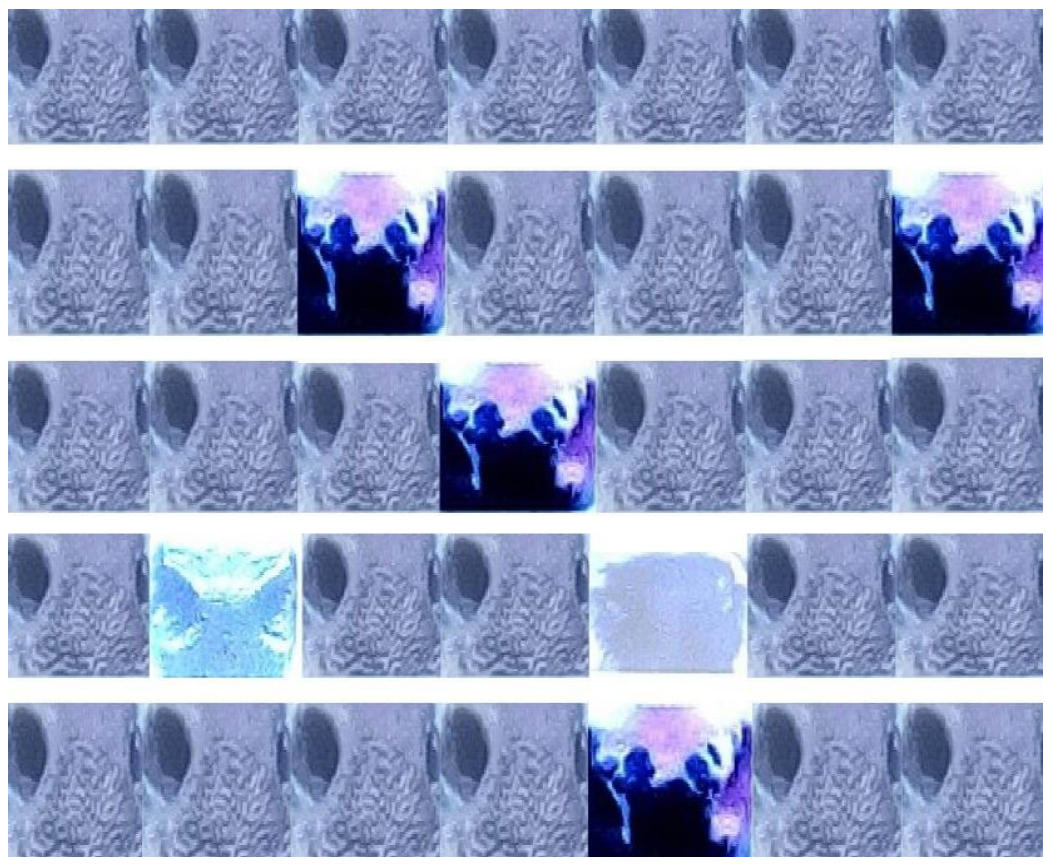

Figure 6. Revealing $17 \%$ corrupted cow nose images using SDAE
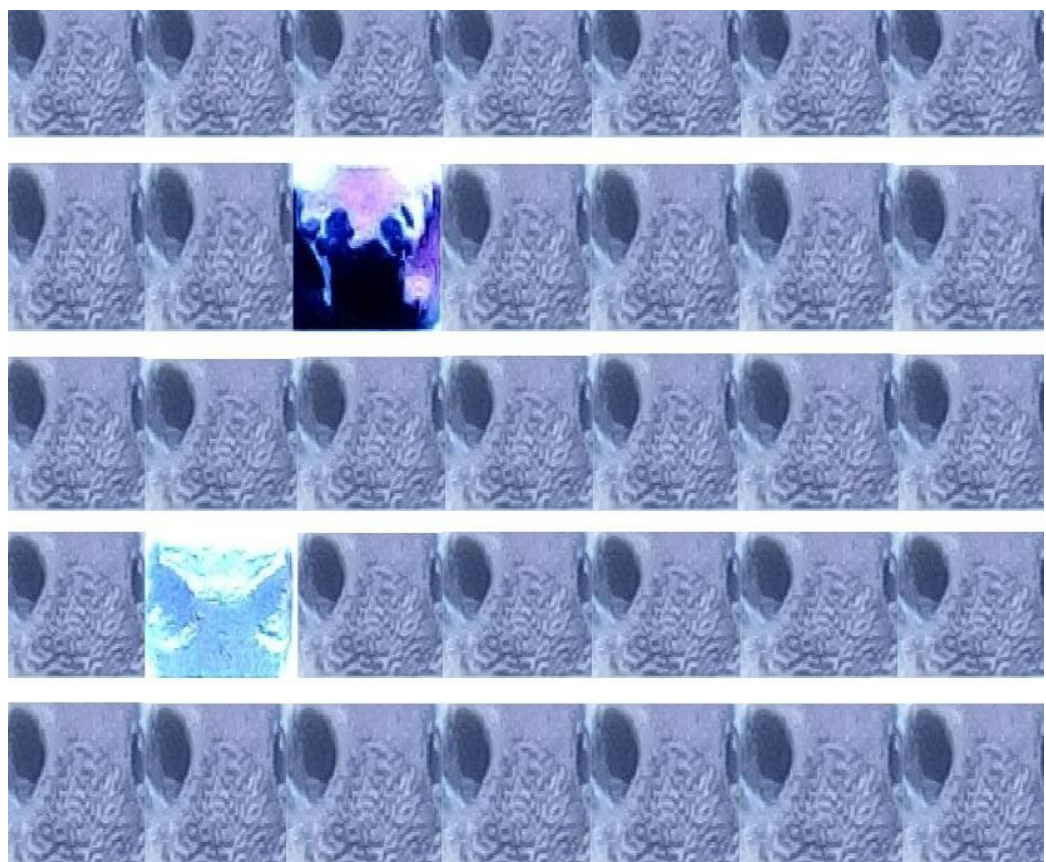

Figure 7. Revealing $5.7 \%$ corrupted cow nose images using other methods 
The accuracy of identification of the two frameworks of deep learning increases with every increase in the size of each patch of the cow nose images. Robust representation of the features of the cow nose image in the different layers of the proposed framework was provided by deep learning algorithm after selecting 400 feature images from each patch $(200 \times 200$ pixels $)$. The selected discriminatory set of the textural feature of the cow nose image reduces whenever the size of each patch of the cow nose images is reduced.

The logistic regression classifier is for the classification based on the $h(l)$ Equations (5) and (6) which is the DBN learning model last hidden layer. The training of the proposed DBN learning model follows the work of Vincent et al. [30], Bengio [31], and Bengio et al. [32] as illustrated in Figure 8, where the DBN learning model is applied by the construction of multiple RBM models. Stacked on top of layers which consist of multiple nodes per each layer fed into the next layer is the RBM classification. RBM's basic working model framework for cow recognition is to ensure that the DBN deep learning framework serves the purpose of extraction and learning of the cow nose images texture features extracted sets.

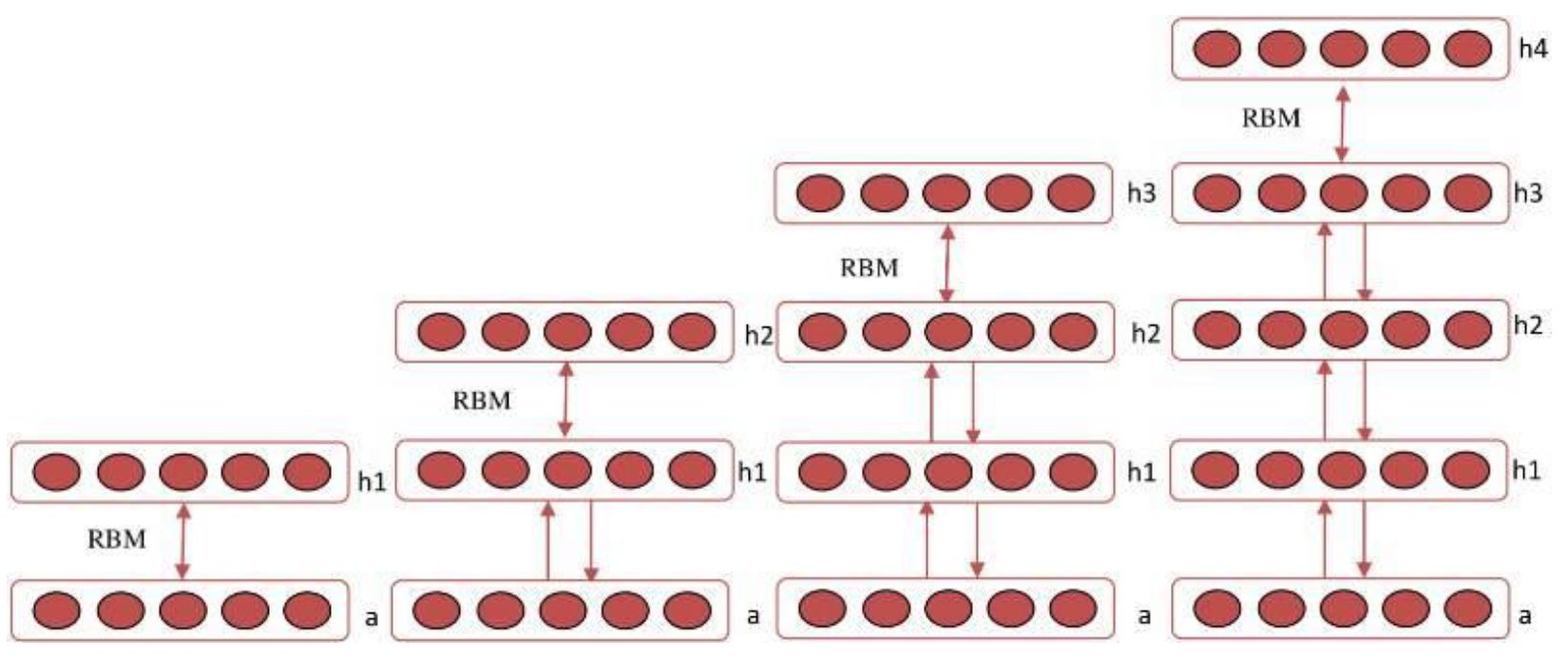

Figure 8. Stacked RBM-based DBN learning model architecture

To compute $\mathrm{R}$-HOG, $\mathrm{m} \times \mathrm{m}$ grids over $\mathrm{n} \times \mathrm{n}$ pixel cells and histogram bins are needed, where $\mathrm{m}$ is the number of cells in each block. Shown in Figure 9 is the R-HOG blocks composed of $3 \times 3$ cells of $6 \times 6$ pixels. Nonetheless, the best configuration is to use $2 \times 2$ cells of $8 \times 8$ pixels with 9 histogram bins. 100 nose images tantamount to $(10$ cows (subject $) \times 10$ images of each subject) were randomly chosen as system training dataset and 300 nose images tantamount to $(30$ cows (subject) $\times 10$ images of each subject) were used as the testing dataset with up to 4 images per subject. The results of the experiment are reported and analyzed as found in Tables 1 and 2.

This simply implies that all the nose image pattern patches have been collectively calculated (as shown in Tables 1 and 2) for the description and representation of the discriminatory set of nose image features to the utmost extent than a set of few patches. Comparing the previous cow recognition techniques proposed by some notable authors among those who are Minagawa et al. [20], it is glaring that the authors did not report the results of the experiment equally owing to the unexplainable filtering techniques. 
Table 1. CNN, SDAE, and DBN deep learning approaches recognition accuracy (\%)

\begin{tabular}{|l|c|l|}
\hline S/N & Proposed techniques & Recognition accuracy (\%) \\
\hline 1 & Convolutional Neural Network & 74.75 \\
\hline 2 & Stacked Denoising Auto Encoder & 85.88 \\
\hline 3 & Deep Belief Network & \\
\hline
\end{tabular}

Table 2. CNN, SDAE, and DBN deep learning approaches recognition accuracy (\%)

\begin{tabular}{|c|c|c|c|}
\hline \multirow{2}{*}{ Number of images } & \multicolumn{2}{|c|}{ Recognition accuracy (\%) } & \\
\cline { 2 - 4 } & $\begin{array}{c}\text { Convolutional Neural } \\
\text { Network }\end{array}$ & $\begin{array}{c}\text { Stacked Denoising Auto } \\
\text { Encoder }\end{array}$ & Deep Belief Network \\
\hline 50 & 61.55 & 62.76 & 63.98 \\
\hline 100 & 64.57 & 65.78 & 66.87 \\
\hline 150 & 68.87 & 68.98 & 72.98 \\
\hline 200 & 72.66 & 72.99 & 74.98 \\
\hline 250 & 73.67 & 74.87 & 76.87 \\
\hline 300 & 77.89 & 78.86 & 80.98 \\
\hline 350 & 79.76 & 79.77 & $\mathbf{9 8 . 9 9}$ \\
\hline 400 & 95.97 & 96.65 & \\
\hline
\end{tabular}

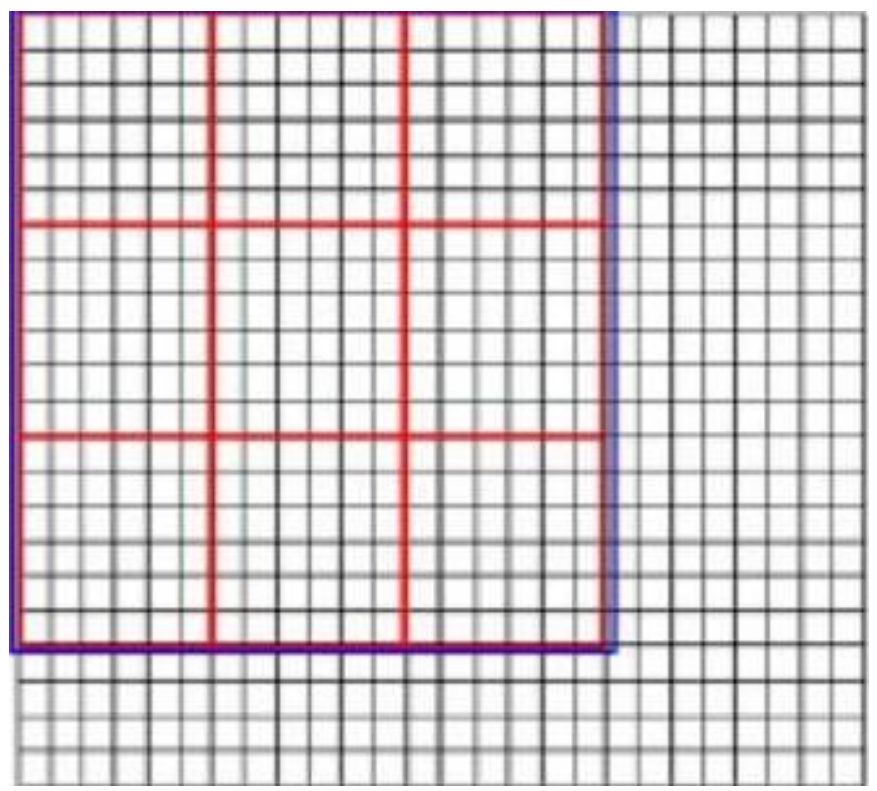

Figure 9. Representation of cells (red), R-HOG (blue) and pixels (black) 


\section{CONCLUSION}

Deep belief network and stacked denoising auto-encoder deep learning-based architectures were proposed in this paper for the recognition and identification of an individual cow using cow nose image pattern. With the approaches used and results generated, DBN is considered as the most suitable on the average. The deep learning approaches were employed to learn a discriminatory feature representation of cow images. The research contributed to the advancement of knowledge in the field of computer vision and pattern recognition as follows: 1) the research digs into new enhanced methods for finding the unparalleled means of individual cow identification using the approaches of deep learning; 2) for the encoding and decoding of the extracted prominent textural features of cow nose images for the recognition of cow, a deep learningbased stacked denoising autoencoder framework was used; 3) stacked denoising auto-encoder and deep belief network were proposed as methods of deep learning for extracting the textural features of cow nose image, and for learning the extracted features for better representation; 4) the employed approaches based on deep learning framework are suited to address the important variations of cow nose images acquired from a poorly lit environment and unstable cow postures resulting to background patches and poor image quality; 5) A multi-layer restricted Boltzmann machine (RBM)-based deep belief network (DBN) technique optimized for cow classification using nose image.

The uniqueness and the changeless textural patterns of the beads and the ridges found on the cow nose image serve as biometrics characteristics for the identification of cow just the same way human fingerprint serves as a unique human identification method. Future work includes real-time identification of animals using their coat patterns.

\section{CONFLICTS OF INTEREST}

No conflict of interest was declared by the authors.

\section{REFERENCES}

[1] Kumar, S., Singh, S.K., Datta, T., Gupta, H.P., "A fast cattle recognition system using smart devices", Proceedings of the 2016 ACM Conference on Multimedia, 742-743, (2016).

[2] Noviyanto, A., Arymurthy, A.M., "Automatic cattle identification based on muzzle photo using speed-up robust features approach", Proceedings of the 3rd European Conference of Computer Science, ECCS, 110:114, (2012).

[3] Kohl, H.S., Burkhart, T., “Animal biometrics: quantifying and detecting phenotypic Appearance”, Trends Ecol. Evol., 28 (7): 432-441, (2013).

[4] Duyck, J., Finn, C., Hutcheon, A., Vera, P., Salas, J., Ravela, S., “Sloop: a pattern retrieval engine for individual animal identification”, Pattern Recogn., 48 (4): 1059-1073, (2015).

[5] Nasirahmadi, A., Richter, U., Hensel, O., Edwards, S., Sturm, B., "Using machine vision for investigation of changes in pig group lying patterns", Computers and Electronics in Agriculture, (119): 184-190, (2015).

[6] Wang, Z., Fu, Z., Chen, W., Hu, J., “A rfid-based traceability system for cattle breeding in china”, in: Proceedings of 2010 IEEE International Conference on Computer Application and System Modeling (ICCASM), (2): V2-567, (2010). 
[7] Krizhevsky, A., Sutskever, I., Hinton, G., "ImageNet classification with deep convolutional neural networks", In Advances in Neural Information Processing Systems, 1097-1105, (2012).

[8] Incetas, M. O., Demirci, R., Yavuzcan, H. G., “Automatic Color Edge Detection with Similarity Transformation”, Gazi University Journal of Science, 32(2): 458-469, (2019).

[9] Sun, Y., Wang, X., Tang, X., "Deep convolutional network cascade for facial point detection", Proc. IEEE Conf. Comput. Vis. Pattern Recognit, 3476-3483, (2013).

[10] Kumar, S., Singh, S.K., "Visual animal biometrics: survey”, IET Biometrics, 6 (3): 139-156, (2016).

[11] Barron, U.G., Butler, F., McDonnell, K., Ward, S., "The end of the identity crisis? Advances in biometric markers for animal identification”, Irish Veterinary J., 62 (3): 204-208, (2009).

[12] Reiter, S., Sattlecker, G., Lidauer, L., Kickinger, F., Öhlschuster, M., Auer, W., ..., Iwersen, M., "Evaluation of an ear-tag-based accelerometer for monitoring rumination in dairy cows", Journal of Dairy Science, 101(4): 3398-3411, (2018).

[13] Seijas, C., Montilla, G., Frassato, L., "Identification of Rodent Species Using Deep Learning", Computación y Sistemas, 23(1): 257, (2019).

[14] Hansen, M.F., Smith, M.L., Smith, L.N., Salter, M.G., Baxter, E.M., Farish, M., Grieve, B., "Towards on-farm pig face recognition using convolutional neural networks", Computers in Industry, 98: 145-152, (2018).

[15] Kumar, S., Singh, S.K., "Cattle Recognition: A New Frontier in Visual Animal Biometrics Research", Proceedings of the National Academy of Sciences, India Section A: Physical Sciences 120, (2019).

[16] Norouzzadeh, M.S., Nguyen, A., Kosmala, M., Swanson, A., Palmer, M.S., Packer, C., Clune, J., "Automatically identifying, counting, and describing wild animals in camera-trap images with deep learning", Proceedings of the National Academy of Sciences, 115(25): E5716-E5725, (2018).

[17] Zin T.T., Phyo, C.N., Tin, P., Hama, H., Kobayashi, I., "Image technology based cow identification system using deep learning", Proceedings of the International MultiConference of Engineers and Computer Scientists 1, (2018).

[18] Kumar, S., Pandey, A., Satwik, K.S.R., Kumar, S., Singh, S.K., Singh, A.K., Mohan, A., "Deep learning framework for recognition of cattle using muzzle point image pattern", Measurement, 116: 1-17, (2018).

[19] Iswanto, I.A., Li, B., "Visual object tracking based on mean-shift and particle-Kalman filter", Procedia Computer Science, 116: 587-595, (2017).

[20] Minagawa, H., Fujimura, T., Ichiyanagi, M., Tanaka, K., Fangquan, M., "Identification of beef cattle by analyzing images of their muzzle patterns lifted on paper", Proceedings of the 3rd Asian Conference for Information Technology in Asian Agricultural Information Technology \& Management, 596-600, (2002). 
[21] Barry, B., Gonzales-Barron, U., McDonnell, K., Butler, F., Ward, S., "Using muzzle pattern recognition as a biometric approach for cattle identification", Trans. ASABE, 50 (3): 1073-1080, (2007).

[22] Dalal, N., Triggs, B., "Histograms of oriented gradients for human detection", Proc. CVPR, 1: 886893, (2005).

[23] Awad, A.I., Zawbaa, H.M., Mahmoud, H.A., Nabi, E.H.H.A., Fayed, R.H., Hassanien, A.E., “A robust cattle identification scheme using muzzle print images", Proceedings of IEEE Federated Conference on Computer Science and Information Systems (FedCSIS), 529-534, (2013).

[24] Noviyanto, A., Arymurthy, A.M., "Beef cattle identification based on muzzle pattern using a matching refinement technique in the sift method", Comp. Electr. Agr., (99): 77-84, (2013).

[25] Kumar S., Tiwari S., Singh S.K., "Face recognition for cattle", Proceedings of $3^{\text {rd }}$ IEEE International Conference on Image Information Processing (ICIIP), 65-72, (2015).

[26] Ehsani, K., Bagherinezhad, H., Redmon, J., Mottaghi, R., Farhadi, A., "Who let the dogs out? modeling dog behavior from visual data", In Proceedings of the IEEE Conference on Computer Vision and Pattern Recognition, 4051- 4060, (2018).

[27] Gaber, T., Tharwat, A., Hassanien, A.E., Snasel, V., "Biometric cattle identification approach based on webers local descriptor and AdaBoost classifier", Comp. Electr. Agr., (122): 55-66, (2016).

[28] Risha, K.P., Chempak, K.A., Sindhu, C.S., "Difference of Gaussian on Frame Differenced Image". International Journal of Innovative Research in Electrical, Electronics, Instrumentation and Control Engineering, 3(1): 92-95, (2016).

[29] Vincent, P., Larochelle, H., Lajoie, I., Bengio, Y., Manzagol, P.-A., "Stacked denoising autoencoders: learning useful representations in a deep network with a local denoising criterion", JMLR (11): 3371-3408, (2010).

[30] Vincent, P., Larochelle, H., Bengio, Y., Manzagol, P.A., "Extracting and composing robust features with denoising autoencoders", Proceedings of the 25th International Conference on Machine Learning, 1096-1103, (2008). ACM.

[31] Bengio, Y., "Learning deep architectures for AI", Foundations and Trends in Machine Learning, 2(1): 1-127, (2009).

[32] Bengio, Y., Courville, A., Vincent, P., "Representation learning: A review and new perspectives," IEEE Transactions on Pattern Analysis and Machine Intelligence, 35(8): 1798-1828, (2013). 